\title{
Integrated care of severe infectious diseases to people with substance use disorders; a systematic review
}

\author{
Jørn Henrik Vold ${ }^{1,2^{*}} \mathbb{D}$, Christer Aas ${ }^{1,2}$, Rafael Alexander Leiva ${ }^{3}$, Peter Vickerman ${ }^{4}$, Fatemeh Chalabianloo ${ }^{1,2}$,
} Else-Marie Løberg ${ }^{1,5,6}$, Kjell Arne Johansson ${ }^{1,2}$ and Lars Thore Fadnes ${ }^{1,2}$

\begin{abstract}
Background: Various integrated care models have been used to improve treatment completion of medications for chronic hepatitis B virus (HBV), chronic hepatitis C virus (HCV), Mycobacterium tuberculosis (TB), and Human immunodeficiency virus (HIV) among people with substance use disorders (SUD). We have conducted a systematic review to evaluate whether integrated models have impacts of the treatment of infectious diseases among marginalized people with SUD.
\end{abstract}

Methods: We searched MEDLINE/PubMed (1946 to 2018, on July 26, 2018) and Embase (from 1974 to 2018, on July $26,2018)$ for randomized controlled trials (RCTs) and cohort studies evaluating diverse integrated models' effects on sustained virological response (SVR), HIV suppression, HBV curation or suppression, completion of TB treatment regimen among people with SUD. The included studies were assessed qualitatively.

Results: Altogether, 1640 studies, and references to 1135 related reviews and RCTs were considered, and only seven RCTs and three cohort studies fulfilled the inclusion criteria. We identified nine integrated care models. Two studies, one RCT and one cohort study, showed a significant effect of their integrated models. The RCT evaluated psychosocial treatment, opioid agonist treatment (OAT) and directly observed TB treatment, and found a significant increase in TB treatment completions among intervention group compared to control group (60\% versus 13\%, $p<$ 0.01). The cohort study including OAT and TB treatments had an effect on TB treatment completion in hospitalized patients ( $89 \%$ versus $73 \%, p=0.03$ ). Eight out of ten studies showed no significant effects of their integrated care models on defined outcomes. One of which having included 363 participants in a RCT showed no effect on SVR compared to the control group when the results adjusted for active substance use and alcohol dependence in a post-hoc analysis ( $11 \%$ versus $7 \%, p=0.49$ ).

Conclusions: The findings indicate uncertainty on the effects of integrated care models' on treatment for severe infectious diseases among people with SUD. Some studies point toward that integrated models could improve care of people with SUD, yet high-quality studies and preferably, sufficiently sized clinical trials are needed to conclude on the degree of impact.

Keywords: Hepatitis C, Human immunodeficiency virus, Mycobacterium tuberculosis, Integrated care, Collaborative care, Substance use disorder, Epidemiology, Systematic review

\footnotetext{
* Correspondence: jorn.vold@uib.no

'Department of Addiction Medicine, Haukeland University Hospital, Bergen,

Norway

2Department of Global Public Health and Primary Care, University of Bergen,

Bergen, Norway

Full list of author information is available at the end of the article
}

(c) The Author(s). 2019 Open Access This article is distributed under the terms of the Creative Commons Attribution 4.0 International License (http://creativecommons.org/licenses/by/4.0/), which permits unrestricted use, distribution, and reproduction in any medium, provided you give appropriate credit to the original author(s) and the source, provide a link to the Creative Commons license, and indicate if changes were made. The Creative Commons Public Domain Dedication waiver (http://creativecommons.org/publicdomain/zero/1.0/) applies to the data made available in this article, unless otherwise stated. 


\section{Background}

Comorbid Hepatitis $\mathrm{C}$ virus (HCV) and hepatitis B virus (HBV), human immunodeficiency virus (HIV), and Mycobacterium tuberculosis (TB) infections are common among people with substance use disorders (SUDs). Due to a low level of attention to personal health care needs, treatment of such severe infectious diseases may be difficult for people who inject drugs (PWID). Additionally, PWID have a sustained high risk of transmitting these severe infections [1-3]. Globally, 1.7 million PWID were estimated to be HIV positive in 2016 [4]. Injection substance use is estimated to account for $1 \%$ of new HBV infections and $23 \%$ of new HCV infections, respectively [5]. A new global strategy to eliminate viral hepatitis that aims to reduce $\mathrm{HCV}$ incidence by $90 \%$ and mortality by 65\% by 2030 was endorsed by the World Health Assembly in 2016 [5]. The World Health Organization (WHO) and the United Nations Sustainable Development Goals (SDGs) have also decided that the global strategies are to end the acquired immune deficiency syndrome (AIDS) epidemic by 2030 and the TB epidemic by 2035 [6-8].To succeed with such ambitious goals, high availability of coordinated care between medical treatment and prevention, psychological care and social-related services for people with SUD is pivotal. A systematic search to identify efficacy of integrated care models on treatment outcomes of severe infectious diseases among people with SUD is required.

Integrated models may improve treatment completion and adherence among people who often are marginalized and hard to reach within current health services systems $[2,3,9,10]$. Comparing the efficacy of these models is therefore of high importance. Different integrated treatment models may improve the access for completing treatment or achieving virus suppression over time but most studies are small and not sufficiently powered to quantify the effect size, and the patterns have been slightly mixed [11-15].

Definitions of the concept of "integrated care" vary. In the hospital setting, WHO defined integrated care as: "an approach to strengthen people-centred health systems through the promotion of the comprehensive delivery of quality services across the life-course, designed according to the multidimensional needs of the population and the individual and delivered by a coordinated multidisciplinary team of providers working across settings and levels of care (...)" [16]. However, this definition is arguably too broad and unspecific, hence, for the purpose of this paper we have chosen to alter the definition of integrated care to "comprehensive set of patient-centered health services that involve the care of chronic infectious disease as a part of coordinated services for people with SUD." Integrate care models and collaborative health services may improve health outcomes to medical care, as well as reduce the burden of disease among people with physical and mental disorders [17], prevent severe infectious diseases [18], improve engagement in TB services [19], and increase patients adherence and staff satisfaction [11]. Systematic reviews suggest that the composition of the integrated models must be described in more detail to identify effective integrated care models that can be implemented in various settings [11, 18-20]. Previous systematic reviews have mostly identified descriptive reports or cohort studies, and only few randomized controlled trials (RCTs) $[11,17,21]$. Also, a number of these studies have methodological limitations such as short follow up period, loss-to-follow-up rate on more than $30 \%$, unclear design and mixed outcome indicators $[11,20]$. In light of the SDGs and the World Health Assembly global strategy for viral hepatitis, TB and AIDS epidemics, a systematic review is imperative to increase knowledge and understanding how to best reach universal coverage of these severe infectious diseases among people with SUD.

In this systematic review, we will examine the effect of integrated health care for people with SUD receiving treatment for HCV, HBV, HIV, and TB. Randomized controlled trials and cohort studies presenting quantitative comparative estimates will be compared to relevant outcomes for each infectious disease. We hypothesized that integrated care models might be useful to reach marginalized low adherent people with SUD, although a high risk of bias and loss-to-follow-up could underpowered the results. This systematic review will summarize the impact of integrated care models on the treatment of severe infectious diseases among people with SUD and will grade the evidence base on methodological quality.

\section{Methods}

\section{Search strategy and selection criteria}

We did a systematic search in MEDLINE/PubMed (1946 to 2018, on July 26, 2018) and Embase (from 1974 to 2018, on July 26, 2018) and Embase online search engines. The search was built up of three main terms; 1) infectious diseases (HCV, HBV, HIV, and TB), 2) substance use disorder and 3) integrated treatment. Sources included MeSH-terms and all components in keywords, title and abstract. The following search terms were employed; 1) infectious diseases: "hepatitis C", OR "hepatitis B", OR "human immunodeficiency virus", OR "tuberculosis", 2) substance use disorder: "substance-related disorder" OR "narcotics agent" OR "withdrawal syndrome" OR "substance abuse" OR "drug" in combination with "dependence", "abuse", "use", "addict" OR "disorder" and, 3) "delivery of health care, integrated" OR "integrated health care system" (Additional files 1 and 2). The search strategy was developed in collaboration with a certified university librarian. 
The three main terms were defined as follow:

1) Severe infectious diseases defined studies where the study population was subject to treatment for $\mathrm{HCV}$, HIV, HBV, or TB.

2) Substance use disorder or behavior was defined as people who use illegal or legal substances, including alcohol, leading to dependency and clinically significant impairment or distress, and being in need of medical treatment in an institution or outpatient clinic for at least one SUD or the complication of the SUD.

3) Integrated treatment among marginalized people with SUD was defined as "comprehensive set of patient-centered health services that involve the care of chronic infectious disease as a part of coordinated services for people with SUD."

WHO has recommended direct observatory therapy (DOT) for TB infected to increase treatment completion and decreased transmission rate [3]. However, for the purpose of this systematic review, and in light of the above definition of integrated care, studies employing solely DOT that was not part of an integrated care model were excluded.

In this paper, only randomized controlled trials and cohort studies were included. Cross-sectional studies, case reports, commentaries, review papers, modeling analysis, and reports without primary data were excluded, as studies lacking control groups. Studies were included for detailed assessment if sub-groups of participants met or were assumed to meet the criteria for substance use disorder. To further validate the literature search, and particularly to ensure that no papers that fulfilled the inclusion criteria were overlooked, we also did an additional search on MEDLINE/PubMed to screen for references of relevant reviews, and RCTs. To ensure that all integrated treatment models were taken into account, we did a broad search, which included MESH-terms from two of the three main terms; substance use disorder and severe infectious diseases (Additional file 1). Then, the relevant RCTs, as well as relevant references and cross-references from the reviews, were selected in line with the inclusion criteria. Studies not including patients with SUD were excluded. The proportion of participants with SUD is given in Table 1.

We employed the Newcastle-Ottawa quality assessment scale (NOS) and the Consolidated Standards of Reporting Trials (CONSORT) 2010 checklist for assessing the quality of the studies. The NOS was graded in eight methodological aspects, and a summary score for selection, comparability, and outcome was ranked in low, medium or high risk of low quality [22]. The CONSORT 2010 checklist was used to assess design, conduct, analysis and interpretation, and the validity of the results [23]. Two independent authors selected included studies, and any discrepancies in the selection process were discussed within a review team of three members to achieve consensus. Duplicate references were removed.

The search was last updated on July 26, 2018. The Preferred Reporting Items for Systematic reviews and Meta-Analysis (PRISMA) criteria were followed throughout the process (Additional file 3) [24].

\section{Outcomes}

Our outcomes were quantitative measures of treatment effect on at least one of the infectious diseases tested in an integrated care model. For HCV, sustained virological response (SVR) at least 12 weeks after the end of treatment was used as a primary outcome. For HIV care, we used HIV viral suppression, and for TB, we evaluated the completion of the recommended treatment regimen. For HBV, we planned to use clearance of HBsAg or HBeAg or suppression of HBV DNA as an indirect measure of intervention effect as outcomes.

\section{Data analysis}

We were prevented from conducting quantitative meta-analysis due to heterogeneity among study populations, incomparability among integrated care models, few comparable studies, and different outcomes measures. Consequently, all studies were assessed qualitatively. All relevant quantitative outcomes of interest were assessed where appropriate. Odds ratio (OR) or relative risk (RR) with $95 \%$ confidence intervals, probability measures $(\mathrm{p}-$ values), and descriptive analyses of outcomes of interest were referred if it was stated in the articles. For comparison, we chose to categorize the included studies into two groups based on whether the participants' received opioid agonist treatment (OAT) or not.

OAT as a multidisciplinary treatment approach has shown to improve HIV treatment and care among PWID [25], and might also serve to influence the effect of integrated care models. Unfortunately, comparability analyses between the groups were impossible due to incomparable studies, and different study populations. In addition, participants with all dependencies were included independent of groups.

\section{Results}

Altogether, 1640 studies were identified in Embase and PubMed, and references to 1135 related reviews and RCTs were considered (Additional file 2). However, only three studies on $\operatorname{HCV}[13,26,27]$, five on $\operatorname{HIV}[14,15$, 28-30], and two on TB [31, 32] fulfilled the inclusion criteria (Fig. 1). Nine integrated models in ten studies were identified, whereas two studies assessed a similar integrated model. We did not identify any relevant 


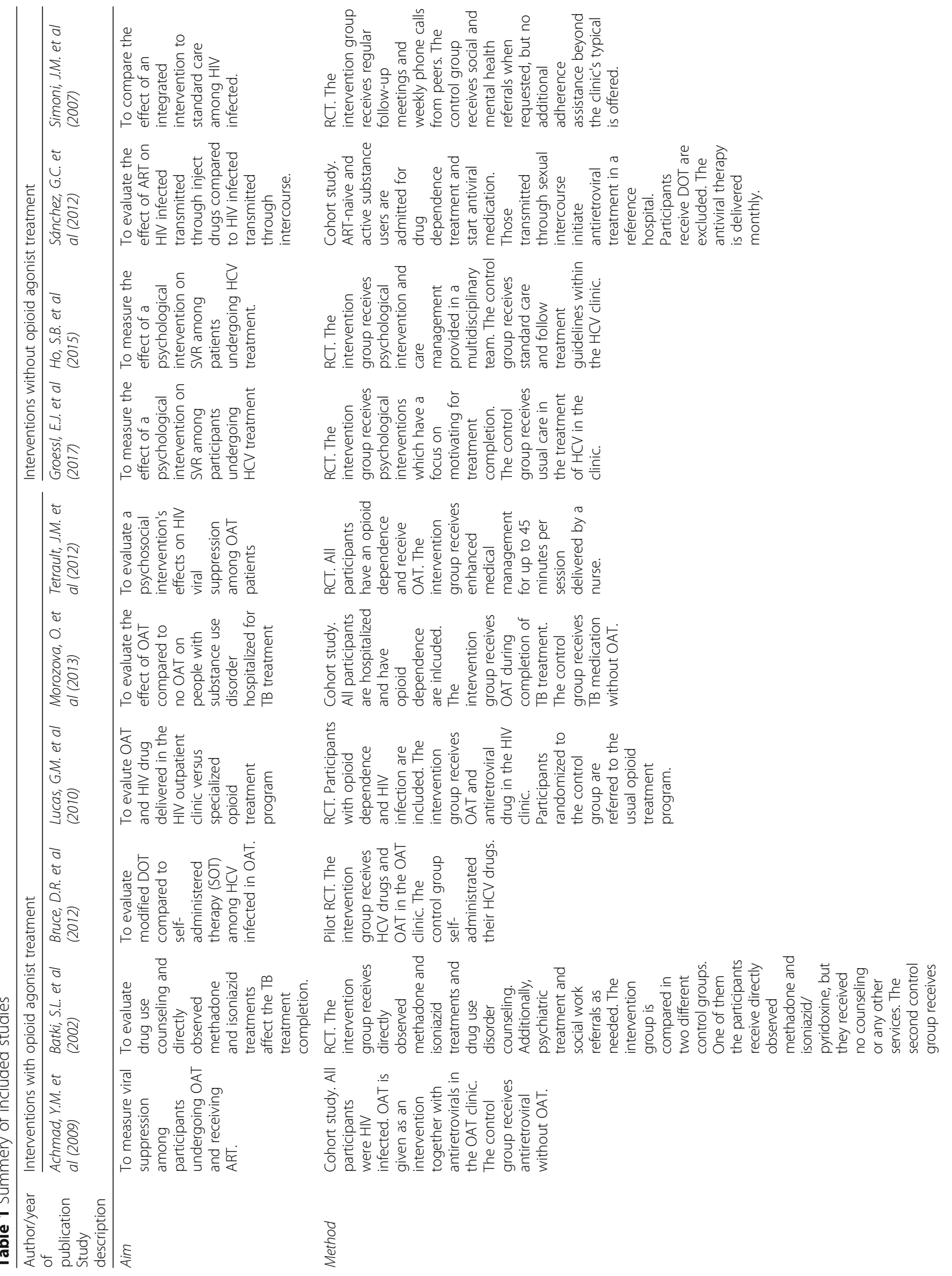




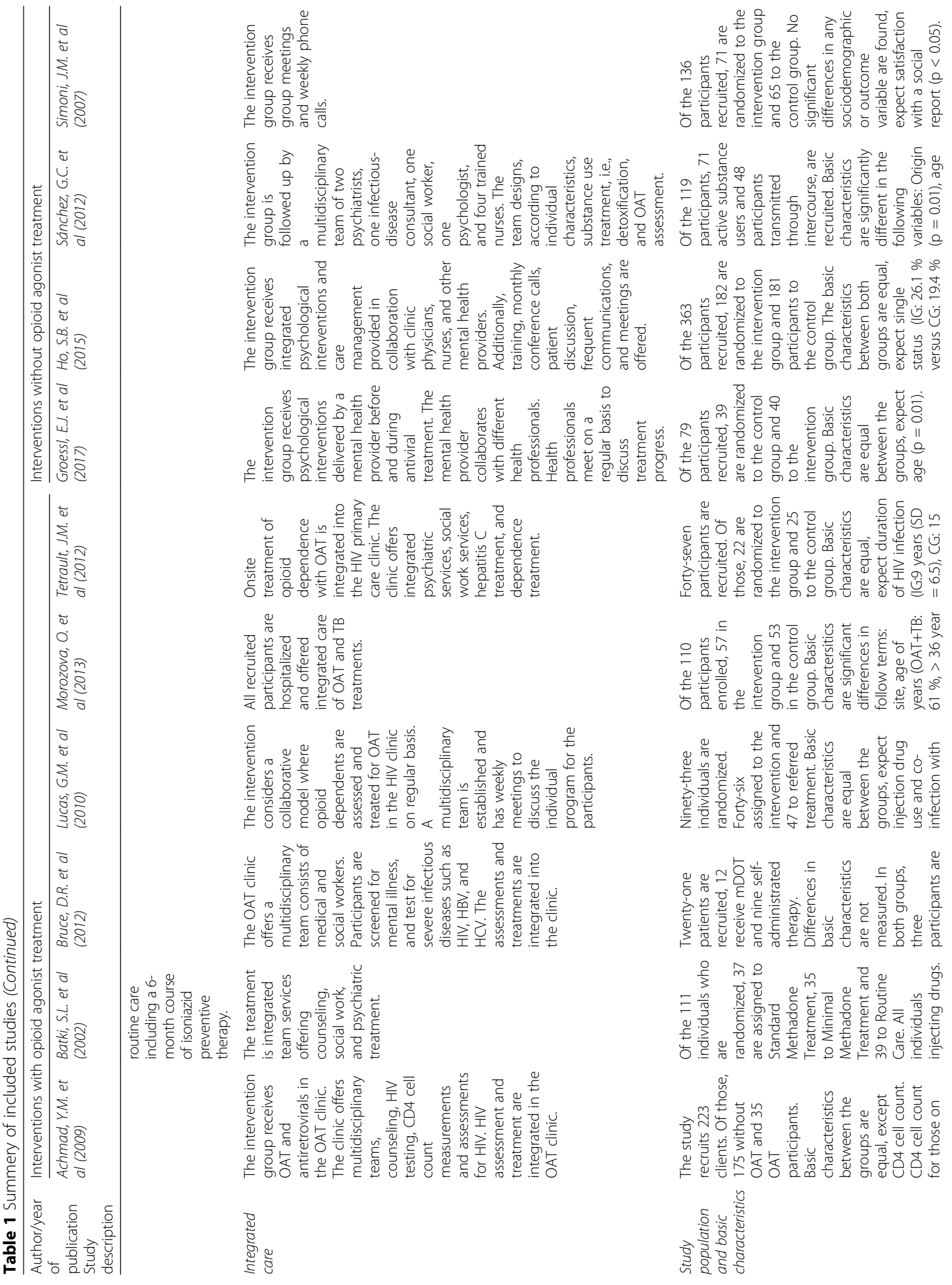




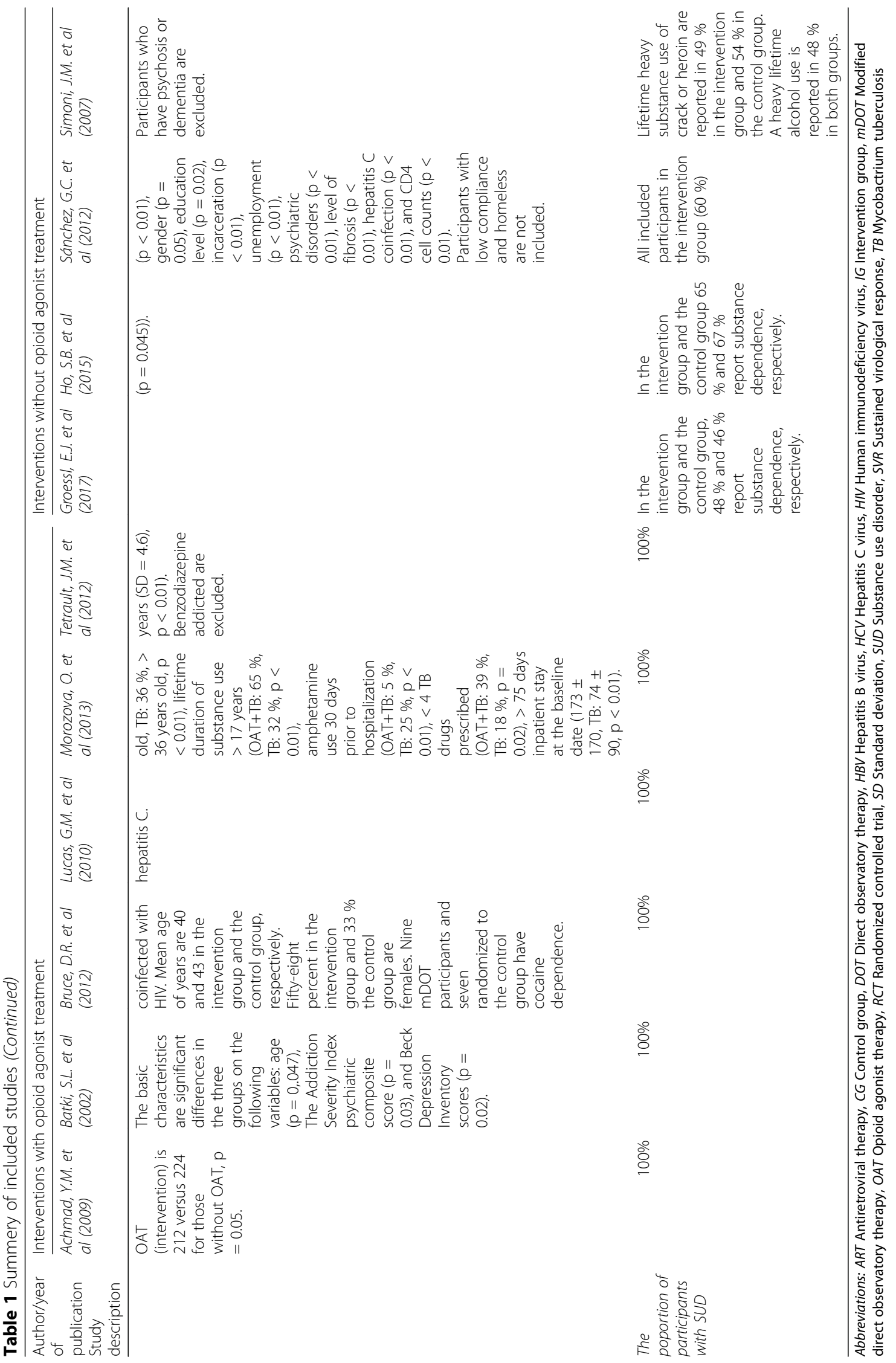




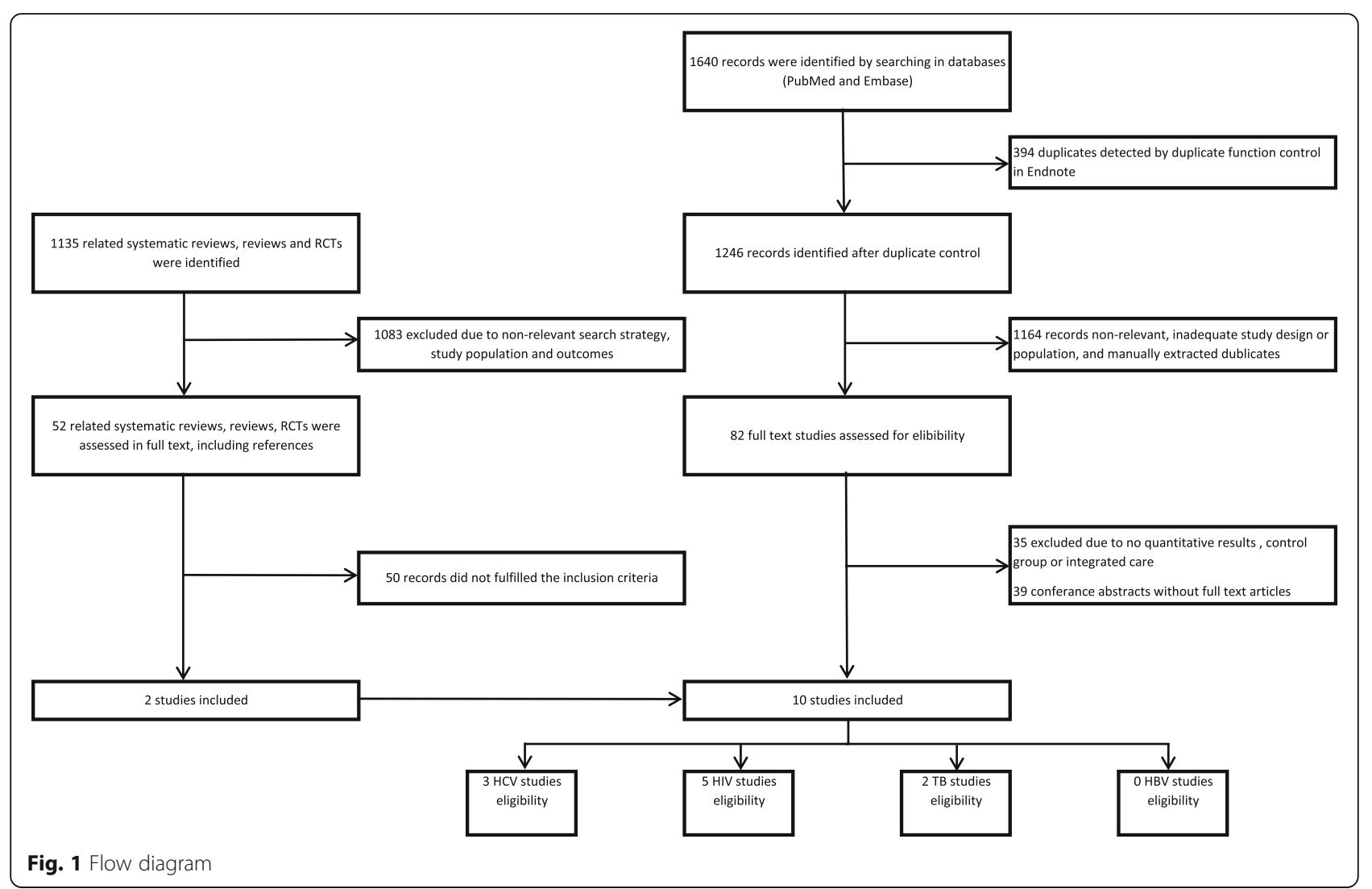

studies on hepatitis B. The CONSORT 2010 checklist or the NOS were employed for assessing the quality of the included studies (Additional files 4 and 5). All included studies assessed integrated models of care when participants underwent medical treatment for $\mathrm{HIV}, \mathrm{HCV}$ or TB. Nine studies recruited patients from either a primary health care centers for people with SUD, OAT outpatient clinics or specialist outpatient clinics for HIV or HCV infected [13-15, 26-30, 32]. One study evaluated TB treatment among hospitalized patients [31]. Four studies also included people without SUD [26-29]. Six out of ten studies were considered to have a loss-to-follow-up rate on more than $30 \%$ (Table 2) [13, $15,26,27,29,32]$.

Different integrated care models were identified. All studies assessed integrated care in multidisciplinary teams or collaborative care teams. Three studies evaluated various psychosocial interventions integrated with treatment of an infectious disease in an OAT program delivery by DOT $[13,31,32]$. Seven studies evaluated the efficacy of brief psychological interventions, regular telephone calls, or social and mental health services in collaboration with other health professionals [14, 15, 26, 28-30,33].

Nine integrated care models were identified, which were subsequently divided into two groups; (1) integrated care with OAT; and (2) integrated care without OAT.

\section{Integrated care and opioid agonist treatment}

Out of the six studies on integrated care with OAT [13$15,30-32]$, only two studies found an effect of the integrated care intervention [31, 32]. Hospitalized patients infected with $\mathrm{TB}$ receiving OAT in an integrated care setting showed a significant improvement on TB treatment completion ( $90 \%$ versus $74 \%, p=0.03$ ) and the adherence to TB medication $(97 \%$ versus $86 \% ; p<0.01)$ after controlling for death and dropouts [31]. Additionally, Batki et al. (2002) found a significant effect on HIV suppression when integrating directly administrated OAT and antiretroviral therapy (ART) against HIV, counseling, psychiatric treatment and social works were compared to standard routine care in an outpatient clinic in people with SUD $(60 \%$ versus $13 \% ; \mathrm{p}<0.01)$ [32]. The remaining four studies showed no significant effect of integrated care on HIV suppression or achievement of HCV SVR when OAT and antiviral therapy against HIV or HCV were integrated at outpatient clinics [13-15, 30].

In the TB study that showed significant effects, integrated care was conducted among hospitalized TB infected [31]. The results indicated that OAT increased the adherence and completion to treatment in a protected environment. The intervention group was recruited from three districts among patients receiving OAT and outpatients TB treatment. Similarity, the 


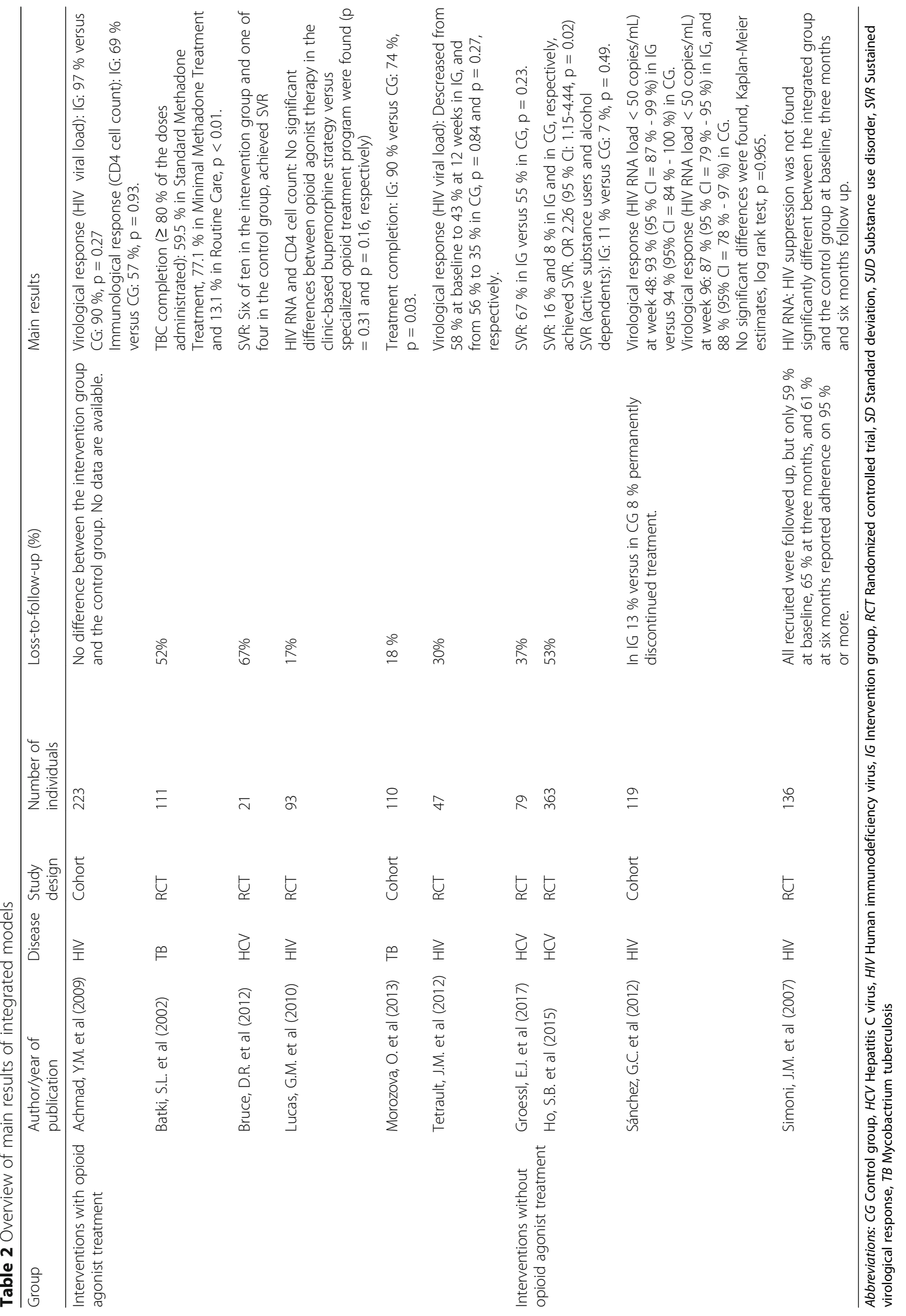


control group was recruited from three districts where people with opioid use disorder were undergoing TB treatment, however, without OAT. Fifty-seven participants were recruited to integrated treatment compared to 53 participants in the control group. DOT was used for TB treatment, and this was integrated with OAT. Basic characteristics were entirely different between the intervention group and the control group, indicating that the study was underpowered or had biases in the randomization. There were differences in the proportion of people above 36 years of age (the intervention group: $61 \%$, the control group: $36 \%, p<0.01$ ), use of amphetamine in the last 30 days (the intervention group: $5 \%$, the control group: $25 \%, \mathrm{p}<0.01$ ), and lifetime duration of substances used more than 17 years (the intervention group: $65 \%$, the control group: $32 \%, \mathrm{p}<0.01)$.

Batki et al. (2002) conducted a randomized controlled trial showing a significant effect on preventive TB treatment completion. TB and OAT treatments were integrated into DOT models with or without psychosocial interventions with 6 months follow-up. TB treatment completion was defined as $80 \%$ or greater of all doses taken. Seventy-seven percent $(n=27)$ of participants randomized into the DOT model without psychosocial interventions achieved treatment completion compared to $60 \%(n=22)$ of participants randomized into DOT model with integrated psychosocial interventions. In the control group, $14 \%(n=5)$ completed the TB treatment. The two OAT treatment groups had significantly higher TB treatment completion rate compared to the control group $(p<0.01)$. However, there were no significant differences between the OAT treatment groups in the rate of TB completion. Characteristics of participants at the baseline were equal between the groups, except age $(p=$ 0.047 ), and scores assessing the severity of problems in individuals with SUD (The Addiction Severity Index psychiatric composite score $(p=0.027)$ and the severity of depression (Beck Depression Inventory scores ( $p=$ $0.022)$ ). Unfortunately, the loss-to-follow-up rate was $52 \%$. The results may indicate a limited effect of psychosocial interventions add to directly administrated therapy to increase treatment completion of TB.

Moreover, a RCT study evaluated a clinic-based integrated treatment model in a HIV clinic [30]. All included participants received antiretroviral therapy in the clinic and had an opioid use disorder. The study evaluated the effect of assessment and initiation of OAT in the HIV clinic on HIV suppression. All opioid addicted who qualified to induct OAT were randomized. Participants randomly assigned to the control group were encouraged to be referred to the opioid treatment program. Participants randomized to the intervention delivered their OAT medications in the HIV clinic during 12 months follow-up. After 12 months, changes from baseline of HIV RNA and CD4 cell counts did not significantly differ between the groups $(p=0.31$ and $p=$ 0.16 , respectively). However, the clinic-based intervention group had higher participation in OAT over 12 months of follow-up. This study enrolled only $78 \%$ of the estimated sample size, which is a limitation of the study's validity. Also, a relatively high loss-to-follow-up rate on $17 \%$ and inclusion of only one single-center impairs the generality of the study's results.

Two more studies evaluated integrated HIV care [14, 15]. HIV suppression and self-reported adherence to ART were evaluated in a non-randomized cohort study that compared integrated HIV treatment in an outpatient clinic among OAT patients to providing ART in an HIV outpatient clinic to patients with SUD [14]. Thirty-five patients were recruited to receive integrated care, and 175 patients recruited to the control group. The virological response was defined as HIV suppression below 400 copies/ $\mathrm{mL}$, and the immunological response as CD4 cell count above 200 cells $/ \mu \mathrm{L}$ after at least 6 months. Among patients enrolled in OAT 97\% achieved virological response compared to $90 \%$ in the control group $(p=0.27)$. Approximately $69 \%$ had an immunological response compared with $57 \%$ in the control group $(p=0.93)$. No significant differences were established among self-reported adherence and virological and immunological response between these groups. Participants in the intervention group received ART weekly and the self-reported number of medications taken the last week was reported. Basic characteristics at baseline and loss-to-follow-up were not significantly different between the groups.

Another randomized controlled trial compared an integrated intervention on HIV suppression among people with opioid use disorder to standard treatment $[15,34]$. Integrated care was psychosocial intervention conducted by nurses. Twenty-two participants were randomized to integrated care and 25 participants received standard care. After the participants were included, and 2 weeks before randomization, all participants were stabilized on buprenorphine-naloxone. The outcome was changes in viral suppression 12 weeks after the intervention was initiated. After 12 weeks the number of HIV copies decreased from $58 \%$ at baseline to $43 \%$ at 12 week in the intervention group, and from 56 to $35 \%$ in the control group, $p=0.84$ and $p=0.27$, respectively, probably due to low sample size. The integrated psychosocial interventions were completed by a mean of 3.0 (standard deviation $(\mathrm{SD})=1.2$ ) sessions per months in 3 months with a mean session length of $47 \mathrm{~min}(\mathrm{SD}=5.3)$. All participants provided consultation with a physician bi-weekly. The adherence to ART and the buprenorphine-naloxone medications was monitored by a medical event monitoring system, micro-electro-mechanical-system (MEMS ${ }^{\bullet}$-cap). Baseline characteristics were equal in both groups, except years of HIV infection among participants (the intervention group: 
$8.7(\mathrm{SD}=6.5)$, the control group: $15.4(\mathrm{SD}=4.6), p<0.01$ ). The loss-to-follow-up was $30 \%$. Some participants received the HIV medication in a different clinical site than that buprenorphine-naloxone was provided, representing a high risk of bias.

A small randomized controlled trial on $\mathrm{HCV}$ evaluated integrated care that included modified DOT compared to self-administrated HCV treatment among patients in OAT [13]. Twenty-one participants were recruited, 12 and nine were randomized to the intervention group and the control group, respectively. The primary outcome was SVR. The modified DOT models were organized in such a way that the hepatitis $\mathrm{C}$ medications and the OAT were taken under observation at the same time. Patients got an injection of peg-Interferon weekly and received the morning doses of Ribavirin together with an opioid agonist medicine. To monitor adherence, participants received a take-home evening doses in $\mathrm{MEMS}^{\oplus}$-cap. Six out of ten and one out of four obtained SVR in the intervention group and the control group, respectively. The loss-to-follow-up was $67 \%$, out of a small initial population. In addition to a prone to selection bias, the results were underpowered to evaluate the effect of the intervention in this study. Small sample size, selection bias, and inconclusive outcomes lead to a careful assessment of the results.

\section{Integrated care without opioid agonist treatment}

We identified four studies with integrated care without OAT [26-29]. Substance use disorders were reported from 46 to $100 \%$ of the participants in the included studies (Table 1). Two of four studies used the terms "substance use disorder", and "substance use", however; the studies did not notice the type of substance dependencies $[26,27]$. These two studies reported that heroin and cocaine were used in 87 and 13\%, and 50 and 52\% of the participants, respectively [28, 29]. One study found a significant effect of brief psychological intervention care having 29 (16\%) participants in the intervention group and $14(8 \%)$ participants in the control group that achieved SVR (OR $=2.26,95 \%$ CI, 1.15-4.44, $p=$ 0.02 ) on patients that were screened and that tested positive for depression, post-traumatic stress disorder (PTSD) and/or substance use disorder. A post-hoc analysis comparing people with and without SUD did not find a significant effect on SVR, having $20(11 \%)$ in the intervention group and $14(7 \%)$ in the control group that achieved SVR, $p=0.49$ ) [27]. Integrated care seems to improve SVR, but as this study was not powered for subgroup analysis of SUD patients the results are not significant and may also have been achieved by change. In contrast, three studies failed to show any effect at all $[26,28,29]$.

A RCT in a HIV primary care outpatient clinic compared an integrated care $(n=65)$ versus standard care $(n=71)$ to
HIV infected [29]. The intervention consisted of six one-hour group session, twice monthly, where treatment barriers, ART adherence, and HIV stigma barriers such as a sexual and romantic relationship, substance use and mental health problems were discussed. The control group received standard care and was given social and mental health referrals. The primary outcome was suppression of HIV RNA at baseline, at 3 and 6 months. A post-hoc analysis revealed no significant differences on HIV suppression between baseline, compared to 3 months and 6 months follow up after treatment initiation. Baseline characteristics were similar in both groups. Only half of the study population had a heavy lifetime substance use of either alcohol, cocaine or heroin. Only 59\% follow-up at baseline, $65 \%$ at 3 months, and $61 \%$ at 6 months, reported taking $95 \%$ or more of their prescribed medications.

Furthermore, a cohort study evaluated HIV suppression between HIV infected transmitted through substance use (intervention) or intercourse (control) followed up with blood samples at baseline, weeks 48 and 96 [28]. Active and recent people with SUD were recruited to the integrated care group where they received medical care, drug use disorder treatment, and psychosocial support. People transmitted through intercourse were received standard medical support at the infectious diseases unit of the reference hospital. The primary outcome of the study was time to loss of virological response defined as HIV RNA above 500 copies $/ \mathrm{mL}$ after getting HIV RNA below 50 copies $/ \mathrm{mL}$, or not getting an HIV RNA below 50 copies $/ \mathrm{mL}$ after week 24 . Seventy-one people transmitted through substance use and 48 matched infected through sexual transmission were included. HIV suppression was measured by HIV RNA. At week 48, 93\% (95\% CI, $87-99 \%)$ of the intervention group and $94 \%$ (95\% CI, $84-100 \%)$ of the control group achieved virus suppression, respectively. At week 96, 87\% (95\% CI, 79 - 95\%) and 88\% (95\% CI, 78 97\%) achieved virus suppression, respectively. No significant differences between substance users and people transmitted through intercourse were found at week 48 $(p=0.13)$ and week $96(p=0.24)$ compared to baseline Kaplan-Meier estimates (log-rank test) of time to loss of virological response and was not significantly different between the groups $(p=0.97)$. Basic characteristics were entirely different such as CD4 cell count (209 cells/ $\mu \mathrm{L}$, range, $3-427$ cells $/ \mu \mathrm{L}$ versus 294 cells $/ \mu \mathrm{L}$, range, $29-465$ cells $/ \mu \mathrm{L}, p<0.01$ ) and psychiatric comorbidities (personality disorder, depression, psychotic disorder, and bipolar disorder, $\mathrm{p}<0.01)$. Loss-to-follow-up was indifferent in the intervention versus control groups, 13 and $8 \%(p=0.18)$, respectively.

Two studies evaluated integrated versus standard $\mathrm{HCV}$ care in a hepatitis $\mathrm{C}$ medical center with $\mathrm{HCV}$ infected veterans with psychiatric diseases or SUD [26, 27]. The 
primary outcome was SVR. Participants randomized to integrated care were seen by mental health providers (one marriage and family therapist and two psychologists) who were educated in cognitive behavioral therapy (CBT) and motivational interviewing (MI). The participants received regular individual appointments, briefing before and under the antiviral treatment. The participants were contacted by the provider monthly and more frequently if they had side effects and worsening of psychiatric illness and substance use disorder during treatment.

In the first randomized HCV cohort study, 363 participants were randomized in total $(n=182$ for integrated care and $n=181$ for standard care) [27]. Of those, 58 in the intervention group and 34 in the standard care group initiated treatment, respectively. In the intervention group, 14 participants achieved SVR (OR 2.26, 95\% CI, 1.15-4.44, $p=0.02$ ). To compare people with and without SUD, a post-hoc analysis on $20(11 \%)$ in the intervention group and 14 (7\%) in the control group was done. No significant effect on SVR was found between these groups, $p=0.49$, but this post-hoc analysis was not powered for this subgroup analysis and the results could be random. Baseline characteristics were equal. Substance use disorder was detected in $66 \%$ of the participants, and $47 \%$ had active substance use. People loss-to-follow-up was $53 \%$.

In the second randomized $\mathrm{HCV}$ cohort study, altogether 79 participants were recruited $(n=40$ in the intervention group and $n=39$ in the control group) [26]. Of those, only 18 and 9 initiated treatment, and 12 and 8 participants achieved SVR $(p=0.23)$, respectively. SVR was not significantly different between these two groups. Both studies used a combination of interferon-based treatment and direct-acting antivirals (DAA). Baseline characteristics were equal, except age (intervention group age $=54.0(\mathrm{SD}$ $=8.7)$ and the control group age $=57.4(\mathrm{SD}=7.7), p=$ $0.01)$. Substance use disorder was identified in only $47 \%$ of the participants, and in $32 \%$ of those using substances actively. Loss-to-follow-up was again considerable at 59.3\%.

Overall, all the included studies, with or without OAT as a part of integrated care model had a high loss-to-follow-up rate $[13,15,26,27,32]$, unclear description of the integrated intervention $[14,31]$, or the primary outcome was based on self-reporting of adherence to medication and substance use $[14,15]$. Five studies had a loss-to-follow rate of more than $30 \%[13,15,26,27,32]$ and for one study the quantitative rate was not given [14]. In this review, one out of four studies without OAT showed significant effects of the interventions. However, this study also included people without SUD [27]. When the results were adjusted for people with SUD or alcohol dependence in a post-hoc analysis, no significant effect on the intervention was found. In total, three out of the ten included studies used psychological interventions as a major part of the study intervention [15, 26, 27]; none of which measured a significant effect on people with SUD compared to the control groups.

\section{Discussion}

We assessed the impact of integrated care models among patients with SUD and severe infectious diseases. Many relevant studies were excluded due to a low level of collaborative care involvement, or measurements of outcomes that were not relevant to our review. Overall, most of the included studies were considered to have a high risk of biases and both the results and methodology were heterogeneous. A recurrent finding was a high loss-to-follow-up rate, unclear description of the interventions, and underpowered studies. Moreover, differences in potential confounders between groups in cohort studies including age, proportion of homelessness, proportion of people who inject drugs, employment status, level of education, and severe psychiatric disorders to name but a few. Potential confounders may further bias the interpretation of the results.

The impact of integrated treatment among people with SUD on viral suppression, HCV SVR and TB treatment completion is uncertain according to our findings. In our review, a quantitative meta-analysis has not been performed due to limited comparable studies of high quality. Only two studies found an effect of integrated treatment. One of them found an effect of integrated OAT given to hospitalized TB-infected patients on treatment completion and medication adherence [31]. The second one found an effect on collaborative care when antivirals and OAT were given as DOT with or without psychosocial interventions [32]. Other OAT integrated models had uncertain effects. A meta-analysis published in 2016 suggested that OAT was associated with an increase in ART coverage and adherence to ART, and limited evidence for OAT decreasing mortality for PWID on ART [25]. A similar impact of OAT on adherence to hepatitis $\mathrm{C}$ and TB treatment has not been shown. However, Altice, FL, et al. (2011) evaluated in a multicenter study an integrated OAT model in a HIV clinical care settings, and found no association between integrated OAT and viral suppression [35]. An important limitation was the lack of a control group, which was the reason why we did not include it in our review.

The adherence to medical treatment in an integrated care model may be influenced by many other factors affecting the patient's ability to follow up treatment as prescribed. Personal characteristics such as age, gender, mobility, lack of motivation, somatic and psychiatric comorbidities, and previous experiences, are some of the examples [36]. OAT as a part of an integrated care model may have an impact on patient's ability and interest to follow up medical treatment. Comprehensively 
integrated care models, including OAT, may consist of different medical, psychological and social approaches influencing the effect on integrated care. The efficacy of these approaches is uncertain and must be explored more in detail in the future RCTs with sufficient power in order to study more precisely the effectiveness of integrated care and how it influences adherence and treatment completion.

Three studies that included psychological intervention by clinical psychologists or psychosocial intervention by nurses under clinical guidance of a clinical psychologist as a part of the integrated care were included in this literature review. The effects of psychological intervention on our outcomes are too weak to measure quantitatively. A previous systematic review has measured the effects of psychosocial interventions on people with SUD and severe mental illnesses quantitatively independently of infectious disease treatment [21]. Different psychosocial interventions were assessed, including MI, CBT, and skills training. Overall, the results were affected by low to very low rate of evidence and unclear risk of bias. Based on 32 RCTs, no compelling evidence to reduce substance use or improve mental state in patients with severe mental illnesses was found. However, a systematic review and meta-analysis on collaborative care models have shown to be useful to enhance mental and physical outcomes among patients with mental disorders [17]. Three studies included people with SUD, but no intervention was qualified to be included as collaborative care models. The main focus of the studies was integrating or coordinating primary care with an ongoing psychosocial oriented substance use treatment program. It is uncertain whether a psychological intervention that is used in the treatment of severe infectious diseases among patients with SUDs improves the viral suppression or treatment completion. Again, there is an urgent need for high-quality RCTs with a detailed description and definition of integrated treatment models effect on quantitatively outcomes measurements, and where the risk of bias is reduced.

Three of the included studies in this systematic review evaluated DOT as a tool to increase treatment completion [13, 31, 32]. Generally, DOT did not meet our definition of integrated care but was included as one of several approaches on three of the integrated care models. In two of the cases, it is uncertain how much the DOT element per se affected the results. In our review, two studies including DOT in an integrated setting showed a significant effect on TB treatment completion. DOT is a controversial method due to challenges with patient's autonomy and could leave the patients as a passive recipient of therapy [37]. However, DOT is a part of the WHO strategy, "Directly Observed Therapy Short course," whose proposing to increase the completion of the TB treatment, and reduce contamination [38]. DOT has been less commonly implemented in HIV, HBV, and
$\mathrm{HCV}$ strategies due to lack of compelling evidence in support for this treatment. DOT has been evaluated in a systematic Cochrane review as a treatment modality to affect treatment completion on TB infected [12]. Interestingly, DOT was compared to self-administration of therapy and compared between PWIDs receiving TB prophylaxis as DOT to standard care. DOT compared to self-administration showed a similar pattern to completion of TB cure, but stratified analyses on people with SUD or marginalized people were not surveyed. Similarity, one study that was included in the Cochrane review compared DOT to standard care among PWIDs [39]. Three hundred PWID received TB prophylaxis delivered by DOT or no observation. Treatment completion was not significantly different between DOT compared to self-administration.

The etiological treatment for hepatitis C and HIV have improved significantly in the last decade. New DAAs against chronic hepatitis $C$ are better tolerated and have a better efficacy than traditional interferon-based treatment, and could therefore markedly reduce the global chronic hepatitis C epidemic [5, 40]. A high cost has limited the distribution to not only low- and middle-income countries, but also high income countries such as Norway [5, 41]. From February 1, 2018, however, all chronic HCV patients in Norway have given an opportunity to receive treatment with DAAs regardless of genotype and liver fibrosis level. Among recent advances in ART against HIV are increased potency making single tablet treatment feasible, better tolerance, and improved viral suppression [42, 43]. In our review, DAAs were not tested systematically in a separate study, and stratified analysis based on this item is not surveyed. However, Norton et al. (2017) have evaluated new DAAs among people who actively use drugs and received OAT in an integrated model [44]. The results of this non-controlled study showed that substance use and OAT are independently associated to a high SVR rate (> 90, 95\% CI: 42 - 99\%) when DAAs are given in a coordinated service. Due to the small sample size and lack of control group, the results are uncertain and must be confirmed in high-quality studies. Further integrated care models and new antivirals may contribute to ensuring better availability and treatment completion.

The chief limitation of this review is the lack of relevant studies, which fulfilled our inclusion criteria. Thus, we were unable to conduct a meta-analysis. Heterogeneity of these studies and methodological shortcomings also made it more challenging to synthesize clear patterns of evidence. In an attempt to minimize methodological shortcomings, we opted a strict definition for inclusion. A wider definition of search strategy will include a wider range of approaches but also approaches where the health care is not substantially different from 
more traditional given health care. This could make it more difficult to assess meaningful effects from comparisons. Thus, trying to balance these aspects we have opted for slightly narrower definition of integrated care than Haldane et al. (2017) have used in a review focusing on HIV, substance use and service integration [11]. Nevertheless, integrated treatment, as well as substance use disorder, are frequently used terms that are used with a range of definitions. Several synonyms and terms applied in the literature, makes it challenging to create a search strategy that identify all terms and synonyms used without using extremely broad search strategies. The additional pragmatic search on references and cross-references of relevant reviews, and RCTs, including two more relevant studies, is a high proportion of studies compared to those identified in the main search. Still, we cannot rule out that some studies lack. Integrated treatment can be seen as a continuous scale from studies comparing models with limited differences from conventional strategies to the studies with pronounced differences. Choosing where to set the exact threshold of the "borderline" studies with limited differences can be difficult. However, the studies with limited differences between more conventional and integrated are unlikely to inform as much on the effects of integrated treatment as studies with more pronounced differences.

Furthermore, one could argue that RCTs and cohort studies measuring other outcomes than HIV viral suppression, HCV SVR and TB treatment completions should be included for a wider range of integrated models. On the other hand, subjective outcomes such as self-reported adherence to treatment approaches, change of substance use, and as well as self-reported change of risk behavior are substantiality uncertain outcomes, which are affected by weaknesses. These risks are potentially more pronounced among people who actively use drugs than others due to the drugs effect on cognitive functions [45]. Due to these argumentations, in this review we want to identify all high-quality studies with hard outcomes on at least one of the infectious diseases.

Finally, one could argue that studies assessing non-SUD patients groups could be included in the assessment, but that could further add to the heterogeneity, and it would be uncertain to what extent the findings could be generalized to people with SUD.

\section{Conclusion}

This systematic review identified various integrated care models to reach and treat a group of marginalized patients with substance use disorders and severe infectious diseases. Even most of the included studies had high risks of biases and presented uncertain results. Hospitalized patients, who received integrated treatment for opioid use disorder during $\mathrm{TB}$ treatment, seemed to improve medication adherence and treatment completion. Additionally, integrated psychosocial interventions and directly observed OAT together with ART have been shown to improve the HIV viral suppression. For chronic HCV infected, no studies yet have shown compelling evidence of its benefits.

\section{Additional files}

Additional file 1: Comprehensive details of the search strategy. (PDF 274 kb)

Additional file 2: Assessed articles, references and cross-references, excluded duplicates. (PDF $1228 \mathrm{~kb}$ )

Additional file 3: PRISMA Checklist 2009. (PDF 424 kb)

Additional file 4: CONSORT 2010. Evaluation of design, conduct, analysis, and interpretation, and the validity of the results in included RCTs. (PDF $357 \mathrm{~kb}$ )

Additional file 5: Newcastle-Ottawa scale. Assessment of the quality of included cohort studies. (PDF 427 kb)

\section{Abbreviations}

AIDS: Acquired immune deficiency syndrome; AOR: Adjusted odds ratio; ART: Antiretroviral therapy; CBT: Cognitive behavioral therapy;

CCM: Collaborative care model; CONSORT: Consolidated Standards of Reporting Trials; DAA: Direct-acting antivirals; DNA: Deoxyribonucleic acid; DOT: Direct observatory therapy; HBeAg: Hepatitis Be antigen; HBsAg: Hepatitis B surface antigen; HBV: Hepatitis B virus; HCV: Hepatitis C virus; HIV: Human immunodeficiency virus; MEMS ${ }^{\circledR}$ : Micro-Electro-MechanicalSystem; Ml: Motivational interviewing; NOS: Newcastle Ottawa score; OAT: Opioid agonist treatment; OR: Odds ratio; PRISMA: Preferred Reporting Items for Systematic reviews and Meta-Analysis; PWID: People who inject drug; RNA: Ribonucleic acid; RR: Relative risk; SUD: Substance use disorder; SVR: Sustained virological response; TB: Mycobacterium tuberculosis; WHO: World Health Organization

\section{Acknowledgments}

We thank university librarian Elisabeth Ebner, Christian Løvold Storhaug, MD, and Christian Ohldieck, MD, for usefully input and contributions in the review process.

\section{Funding}

The study was funded by The Norwegian Research Council (BEHANDLING, contract no 269855) and the Western Norway Regional Health Authority («Åpen prosjektstøtte») with Department of Addiction Medicine, Haukeland University Hospital as responsible institution. The funders had no role in study design, data collection and analysis, decision to publish, or preparation of the manuscript. Two of the authors are funded from the research grant BEHANDLING related to the project INTRO-HCV from the Norwegian Research Council. Their respective affiliations fund the other authors.

\section{Availability of data and materials}

Supplemental tables and data sources in this systematic review are available in this published article and its additional file.

\section{Authors' contributions}

$J H V$ was involved in led the study design, coordinated the literature search, extracted all relevant data, analysis, article preparation, and article review. CA contributed in the literature search, extracted relevant data, and article review. LTF and KAJ were involved in study design, supervision of literature search and extraction, analysis and article preparation. RAL, PV, EL, and FC were contributed in interpretation of analysis and writing the article preparation, and article review. All authors have read and approved the final article.

Ethics approval and consent to participate Not applicable. 


\section{Consent for publication}

Not applicable.

\section{Competing interests}

The authors declare that they have no competing interests.

\section{Publisher's Note}

Springer Nature remains neutral with regard to jurisdictional claims in published maps and institutional affiliations.

\section{Author details}

'Department of Addiction Medicine, Haukeland University Hospital, Bergen, Norway. ${ }^{2}$ Department of Global Public Health and Primary Care, University of Bergen, Bergen, Norway. ${ }^{3}$ Department of Internal Medicine, Haukeland University Hospital, Bergen, Norway. ${ }^{4}$ University of Bristol, Bristol, UK. ${ }^{5}$ Division of Psychiatry, Haukeland University Hospital, Bergen, Norway. ${ }^{6}$ Department of Clinical Psychology, University of Bergen, Bergen, Norway.

Received: 29 September 2018 Accepted: 19 March 2019 Published online: 04 April 2019

\section{References}

1. UNAIDS: UNAIDS report on the global AIDS epidemic, 2012. 2012.

2. Walsh N, Verster A, Rodolph M, Akl EA. WHO guidance on the prevention of viral hepatitis B and C among people WHO inject drugs. Int J Drug Policy. 2014;25(3):363-71.

3. Policy guidelines for collaborative TB and HIV services for injecting and other drug users - an integrated approach. In.: World Health Organization, United Nations Office on Drugs and Crime, and UNAIDS; 2008.

4. Consolidated guidelines on HIV prevention, diagnosis, treatment and care for key populations - 2016 update. In. https://www.who.int/hiv/pub/ guidelines/keypopulations-2016/en/: World Health Organization; 2014.

5. Global Hepatitis Report, 2017. In. https://www.who.int/hepatitis/ publications/global-hepatitis-report2017/en/: World Health Organization; 2017: 83.

6. Global health sector strategy on HIV, 2016-2021. In.. http://www.who.int/hiv/ strategy2016-2021/ghss-hiv/en/; World Health Organization; 2016.

7. The End TB Strategy - global strategy and targets for tuberculosis prevention, care and control after 2015. In. . http://www.who.int/tb/ strategy/End_TB_Strategy.pdf?ua=1 World Health Organization; 2014.

8. Good Health And Well-being: Why It Matters. In. https://www.un.org/ sustainabledevelopment/wp-content/uploads/2017/03/ENGLISH_Why_it_ Matters_Goal_3_Health.pdf: United Nations Sustainable Development Goals; 2018: Goal 3.

9. Schechter MT, Strathdee SA, Cornelisse PG, Currie S, Patrick DM, Rekart ML, O'Shaughnessy MV. Do needle exchange programmes increase the spread of HIV among injection drug users?: an investigation of the Vancouver outbreak. AIDS (London, England). 1999;13(6):F45-51.

10. Primary Health Care - Now More Than Ever. In.: World Health Organization; 2008.

11. Haldane V, Cervero-Liceras F, Chuah FL, Ong SE, Murphy G, Sigfrid L, Watt N, Balabanova D, Hogarth S, Maimaris W, et al. Integrating HIV and substance use services: a systematic review. J Int AIDS Soc. 2017;20(1):21585.

12. Karumbi J, Garner P: Directly observed therapy for treating tuberculosis. Cochrane Database Syst Rev 2015, 2015 (5) (no pagination)(CD003343).

13. Bruce RD, Eiserman J, Acosta A, Gote C, Lim JK, Altice FL. Developing a modified directly observed therapy intervention for hepatitis $C$ treatment in a methadone maintenance program: implications for program replication. Am J Drug Alcohol Abuse. 2012;38(3):206-12.

14. Achmad $Y M$, Istiqomah $A N$, Iskandar $S$, Wisaksana $R$, van Crevel $R$, Hidayat $T$. Integration of methadone maintenance treatment and HIV care for injecting drug users: a cohort study in Bandung, Indonesia. Acta Med Indones. 2009; 41(Suppl 1):23-7.

15. Tetrault JM, Moore BA, Barry DT, O'Connor PG, Schottenfeld R, Fiellin DA, Fiellin LE. Brief versus extended counseling along with buprenorphine/ naloxone for HIV-infected opioid dependent patients. J Subst Abuse Treat. 2012;43(4):433-9.

16. Integrated care models: an overview. In. http://www.euro.who.int/_data/ assets/pdf_file/0005/322475/Integrated-care-models-overview.pdf: World Health Organization; 2016.
17. Woltmann E, Grogan-Kaylor A, Perron B, Georges H, Kilbourne AM, Bauer MS. Comparative effectiveness of collaborative chronic care models for mental health conditions across primary, specialty, and behavioral health care settings: systematic review and meta-analysis. Am J Psychiatry. 2012; 169(8):790-804.

18. Crepaz N, Baack BN, Higa DH, Mullins MM. Effects of integrated interventions on transmission risk and care continuum outcomes in persons living with HIV: Meta-analysis, 1996-2014. Aids. 2015;29(18):2371-83.

19. Grenfell P, Baptista Leite R, Garfein R, de Lussigny S, Platt L, Rhodes T. Tuberculosis, injecting drug use and integrated HIV-TB care: A review of the literature. Drug Alcohol Depend. 2013;129(3):180-209.

20. Bajis S, Dore GJ, Hajarizadeh B, Cunningham EB, Maher L, Grebely J. Interventions to enhance testing, linkage to care and treatment uptake for hepatitis C virus infection among people who inject drugs: A systematic review. Int J Drug Policy. 2017;47:34-46.

21. Hunt GE, Siegfried N, Morley K, Sitharthan T, Cleary M. Psychosocial interventions for people with both severe mental illness and substance misuse. In: The Cochrane database of systematic reviews. 2013/10/05 edn; 2013: Cd001088

22. Wells GA, Shea B, O'Connell D, Peterson J, Welch V, Losos M, Tugwell P: The Newcastle-Ottawa Scale (NOS). In., vol. 2018. http://www.ohri.ca/programs/ clinical_epidemiology/oxford.asp.

23. CONSORT 2010 checklist. In. http://www.consort-statement.org/download/ Media/Default/Downloads/CONSORT\%202010\%20Checklist.doc: CONSORT Consolidated Standards of Reporting Trials; 2010.

24. Moher D, Liberati A, Tetzlaff J, Altman DG. Preferred reporting items for systematic reviews and meta-analyses: the PRISMA statement. J Clin Epidemiol. 2009;62(10):1006-12.

25. Low AJ, Mburu G, Welton NJ, May MT, Davies CF, French C, Turner KM, Looker KJ, Christensen H, McLean S, et al. Impact of Opioid Substitution Therapy on Antiretroviral Therapy Outcomes: A Systematic Review and Meta-Analysis. Clin Infect Dis. 2016;63(8):1094-104.

26. Groessl EJ, Liu L, Sklar M, Ho SB: HCV Integrated Care: A Randomized Trial to Increase Treatment Initiation and SVR with Direct Acting Antivirals. International Journal of Hepatology 2017, 2017 (no pagination)(5834182).

27. Ho SB, Brau N, Cheung R, Liu L, Sanchez C, Sklar M, Phelps TE, Marcus SG, Wasil MM, Tisi A, et al. Integrated Care Increases Treatment and Improves Outcomes of Patients With Chronic Hepatitis C Virus Infection and Psychiatric Illness or Substance Abuse. Clin Gastroenterol Hepatol. 2015; 13(11):2005-2014.e2003.

28. Sanchez GV, Llibre JM, Torrens M, Sanvisens A, Mateu G, Knobel H, Langohr K, Santos JR, Muga R. Effectiveness of antiretroviral therapy in HIV-1-infected active drug users attended in a drug abuse outpatient treatment facility providing a multidisciplinary care strategy. Curr HIV Res. 2012;10(4):356-63.

29. Simoni JM, Pantalone DW, Plummer MD, Huang B. A randomized controlled trial of a peer support intervention targeting antiretroviral medication adherence and depressive symptomatology in HIV-positive men and women. Health Psychol. 2007;26(4):488-95.

30. Lucas GM, Chaudhry A, Hsu J, Woodson T, Lau B, Olsen Y, Keruly JC, Fiellin DA, Finkelstein R, Barditch-Crovo P, et al. Clinic-based treatment of opioiddependent HIV-infected patients versus referral to an opioid treatment program: A randomized trial. Ann Intern Med. 2010;152(11):704-11.

31. Morozova O, Dvoryak S, Altice FL. Methadone treatment improves tuberculosis treatment among hospitalized opioid dependent patients in Ukraine. Int J Drug Policy. 2013;24(6):e91-8.

32. Batki SL, Gruber VA, Bradley JM, Bradley M, Delucchi K. A controlled trial of methadone treatment combined with directly observed isoniazid for tuberculosis prevention in injection drug users. Drug Alcohol Depend. 2002; 66(3):283-93.

33. Ho SB, Groessl EJ, Weingart KR, Ward M, Sklar M, Robinson S, Johnson N, Phelps TE, Marcus S, Cheung R, et al. Prospective multicenter evaluation of hepatitis $C$ patients for psychiatric/substance use risk factors that reduce likelihood of antiviral treatment. Hepatology. 2011;54:1194A-5A.

34. Sorensen $\mathrm{J}$, Mascovich A, Wall TL, DePhilippis D, Batki SL, Chesney M. Medication adherence strategies for drug abusers with HIV/AIDS. AIDS Care. 1998;10(3):297-312

35. Altice FL, Bruce RD, Lucas GM, Lum PJ, Korthuis PT, Flanigan TP, Cunningham CO, Sullivan LE, Vergara-Rodriguez P, Fiellin DA, et al. HIV treatment outcomes among HIV-infected, opioid-dependent patients receiving buprenorphine/ naloxone treatment within HIV clinical care settings: results from a multisite study. J Acquir Immune Defic Syndr. 2011;56(Suppl 1):S22-32. 
36. Munro SA, Lewin SA, Smith HJ, Engel ME, Fretheim A, Volmink J. Patient adherence to tuberculosis treatment: a systematic review of qualitative research. PLOS Med. 2007;4(7):e238.

37. Zwarenstein M, Schoeman JH, Vundule C, Lombard CJ, Tatley M. Randomised controlled trial of self-supervised and directly observed treatment of tuberculosis. Lancet (London, England). 1998;352(9137):1340-3.

38. An Expanded DOTS Framework for Effective Tuberculosis Control. Stop TB Communicable Disease. In. https://apps.who.int/iris/bitstream/handle/10665/ 67232NHO_CDS_TB_2002.297.pdf;jsessionid=

EC1F4973A8C7246C716B7580BD0557DE? sequence $=1$ : World Health Organization; 2002.

39. Chaisson RE, Barnes GL, Hackman J, Watkinson L, Kimbrough L, Metha S, Cavalcante S, Moore RD. A randomized, controlled trial of interventions to improve adherence to isoniazid therapy to prevent tuberculosis in injection drug users. Am J Med. 2001;110(8):610-5.

40. Progress report on access to hepatitis $C$ treatment - focus on overcoming barriers in low- and middle-income countries. In. http://apps.who.int/iris/ bitstream/handle/10665/260445/WHO-CDS-HIV-18.4-eng.pdf;jsessionid= 192D3F16578C963DA7651426EE028132? sequence $=1$ : World Health Organization; 2018.

41. Wisloff T, White R, Dalgard O, Amundsen EJ, Meijerink H, Lovlie AL, Klovstad H. Economic Evaluation of Direct-Acting Antivirals for Hepatitis C in Norway. PharmacoEconomics. 2018;36(5):591-601.

42. Kanters S, Vitoria M, Doherty M, Socias ME, Ford N, Forrest Jl, Popoff E, Bansback N, Nsanzimana S, Thorlund K, et al. Comparative efficacy and safety of first-line antiretroviral therapy for the treatment of HIV infection: a systematic review and network meta-analysis. The lancet HIV. 2016;3(11): e510-20.

43. Consolidated guidelines on the use of antiretroviral drugs for treating and preventing HIV infection. In., 2nd edn: World Health Organization; 2016.

44. Norton BL, Fleming J, Bachhuber MA, Steinman M, DeLuca J, Cunningham CO, Johnson N, Laraque F, Litwin AH. High HCV cure rates for people who use drugs treated with direct acting antiviral therapy at an urban primary care clinic. Int J Drug Policy. 2017;47:196-201.

45. Berghaus G, Sticht G, Grellner W, Lenz D, Naumann T, Wiesenmülle S. Metaanalysis of empirical studies concerining the effects of medicines and illegal drugs including pharmacokinetics on safe driving. Driving under the influence of drugs, alcohol, and medicines; 2011.

Ready to submit your research? Choose BMC and benefit from:

- fast, convenient online submission

- thorough peer review by experienced researchers in your field

- rapid publication on acceptance

- support for research data, including large and complex data types

- gold Open Access which fosters wider collaboration and increased citations

- maximum visibility for your research: over $100 \mathrm{M}$ website views per year

At $\mathrm{BMC}$, research is always in progress.

Learn more biomedcentral.com/submissions 